\title{
АВТОМАТИЗИРОВАННЫЙ АЛГОРИТМ ОЦЕНКИ МАРКЕТИНГОВОЙ НАДЕЖНОСТИ ИННОВАЦИОННЫХ РЕШЕНИЙ
}

\author{
(c) 2021 Родионов Дмитрий Григорьевич \\ доктор экономических наук, профессор, Высшая инженерно-экономическая школа \\ Санкт-Петербургский политехнический университет Петра Великого, Россия, Санкт-Петербург \\ E-mail: dmitry.rodionov@spbstu.ru \\ (C) 2021 Куракин Владимир Иванович \\ соискатель, Высшая инженерно-экономическая школа \\ Санкт-Петербургский политехнический университет Петра Великого, Россия, Санкт-Петербург \\ E-mail:v.i.kurakin@yandex.ru
}

\section{(c) 2021 Конников Евгений Александрович}

кандидат экономических наук, доцент, Высшая инженерно-экономическая школа

Санкт-Петербургский политехнический университет Петра Великого, Россия, Санкт-Петербург

E-mail: konnikov.evgeniy@gmail.com

Процесс цифровизации всех экономических процессов на данный момент значительно ускорился, что во многом является следствием пандемии COVID-19. Данный процесс способствую насыщению информационной среды, что в свою очередь приводит к повышению доступности специализированной информации. Ранее уникальный опыт экспертов в той или иной области был крайне значим и ценился на рынке, в то время как сейчас данный опыт может быть следствием углубленного изучения информационной среды исследуемого объекта. В тоже время, данная информация сложно квантифицируема, так как представлена как правило в естественной форме. В рамках статьи [1] авторами был предложен инструментарий квантификации информации, описывающей в цифровой среде инновационные решения, для целей сравнения их маркетинговой надежности. В рамках данной статьи приводятся результаты алгоритмизации и автоматизации данного инструментария.

Ключевые слова: маркетинговая надежность, нефтепромысловое оборудование, лизинг, инновационные решения, информационная среда.

В рамках статьи [1] авторами был предложен инструментарий оценки маркетинговой надежности инновационного решения, позволяющий проводить объективный сравнительный анализ способности исследуемых инновационных решений сохранять во времени в установленных пределах значения параметров, характеризующих способность удовлетворять потребности потенциальных потребителей. Объективность оценки во многом достигается за счет полного или частичного исключения из процесса оценки экспертной составляющей, замещенной значимыми объемами объективной информации, представленной в естественной форме и соответствующей свойствам «Big-Data» массива. Данная специфика определяет возможность автоматизации процесса оценки маркетинговой надежности инновационного решение. Для целей универсализации рассматриваемого автоматизированного алгоритма определена необходимость изначальной идентификации инновационных решений в рамках отраслевого контекста, что частично трансформирует представленный ранее алгоритм.

В рамках первичного этапа реализации описываемого автоматизированного алгоритма осуществляется определение ключевых инновационных решений в рамках отраслевого контекста. Источником данной информации может выступать агрегатор исследовательских результатов, которые значимо представлены в цифровой среде. Одним из наиболее значимых агрегаторов исследовательских результатов выступает цифровой ресурс «ScienceDirect». Данный ресурс был запущен в марте 1997 года, его собственником является издательство Elsevier и на данный момент на нем представлено содержание 2500 научных журналов и 26000 электронных книг 
[2]. Ключевыми особенностями данного ресурса являются бесплатный доступ к краткому содержанию исследований, а также возможность многомерного поиска, что определяет возможность конкретизации исследовательского запроса. Архитектура электронного ресурса позволяет формировать URL запроса в соответствии с требуемой спецификой, следствием чего является возможность использования инструментов автоматизированного скрэпинга. Для данных целей была выбрана библиотека Selenium, позволяющая средствами языка программирования Python автоматизировать процесс извлечения информации, описывающей результаты исследований в рамках рассматриваемой сферы. В качестве элементов данного информационного массива выступают заголовки статей, определенные алгоритмами ресурса «ScienceDirect» в рамках сформированного запроса. Объективным ограничением в данном случае выступает установленное «ScienceDirect» количество выдаваемых результатов, которое составляет 1000 заголовков. Автоматизированный процесс извлечения данной информации представлен на рисунке 1.

По результатам реализации приведенного алгоритма формируется единый, хронологически упорядоченный датафрейм, содержащий до 1000 заголовков статей, описывающих результаты научных исследований в рамках исследуемой области. Для целей идентификации тематических направлений исследований необходима предварительное исключение из сформированного массива низкосодержательных лексем, а также приведения содержательных лексем к единой базовой словарной форме. Данный процесс может быть назван токенизацией сформированного массива естественной информации. Данный процесс предполагает последовательную реализацию следующих этапов:

1. Первичная токеннизация. В рамках данного этапа входной массив информации дифференцируется на элементарные лексемы, после чего производится исключение пунктуационных и вспомогательных лексем, а также приведение оставшихся лексем к единому регистру.

2. Морфологическая разметка первичного массива токенов. В рамках данного этапа производится определение части речи каждого из токенов первичного массива, по результатам чего исключаются все токена, не являющиеся прилагательными, существительными или глаголами.

3. Лемматизация морфологически размеченного массива токенов. В рамках данного этапа производится приведение сформированного ранее массива существительных, прилагательных и глаголов к их базовой словарной форме (лемматизация), и формирование единого массива лемматизированных токенов.

4. Обработка низкосодержательных лексем. На данном этапе осуществляется исключение из сформированного массива низкосодержательных лексем, в соответствии с универсальным или индивидуально сформированным массивом.

По результатам реализации представленного алгоритма формируется результирующий массив содержательных токенов, на основе которого могут быть идентифицированы основные направления инновационных решений в рам-

1) Установка необходимых инструментальных библиотек:

- $\quad$ selenium - совокупность инструментов автоматизированного извлечения цифровой информации.

- bs4 - совокупность инструментов структурирования HTML и CSS разметки.

- $\quad$ time - совокупность инструментов управления временем потока.

2) Определение входных настроек

Webdriver: $\quad$ options = webdriver.ChromeOptions()

- browser - инструмент обращения 5 options.add_argument("--incognito")

к веб-браузеру.

browser $=$ webdriver.Chrome (executable_path $=$ Путь $\mathrm{k}$ chromedriver , chrome_options = options)

3) Извлечение информационного массива:

- $\quad$ titles - формируемый список заголовков статей.

- url-трансформируемый цифровой запрос.

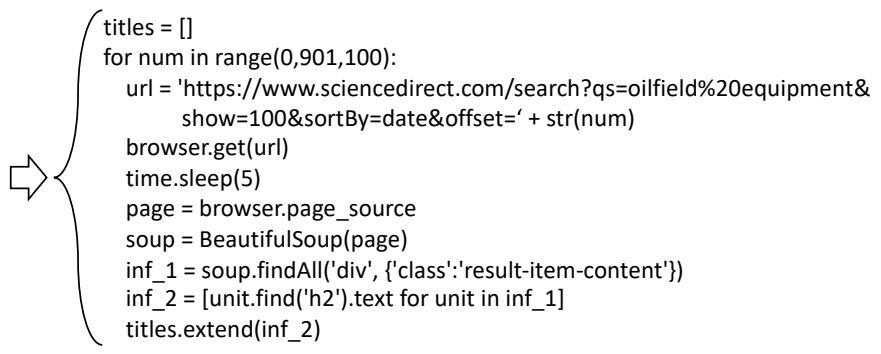

Рисунок 1. Автоматизированный алгоритм извлечения информации из ресурса «ScienceDirect». 
ках исследуемого контекста. Данный алгоритм может быть назван «воронкой токенизации», а его визуализация представлена на рисунке 2.

Процесс реализации воронки токенизации может быть также автоматизирован средствами Python, в частности посредствам инструментов библиотеки NLTK. Автоматизированный процесс токенизации входного массива текстовой информации представлен на рисунке 3.

Сформированный в результате массив то- кенов в полной мере должен отражать содержательные свойства исследуемой области инновационных решений. На завершающем этапе в данном случае производится идентификация ключевых направлений формирования инновационных решений в рамках исследуемой области. Данный процесс может быть автоматизирован посредствам кластеризация сформированного массива токенов. Процесс кластеризации является одним из видов клас-

Входной массив естественной информации, представленной в текстовой форме.

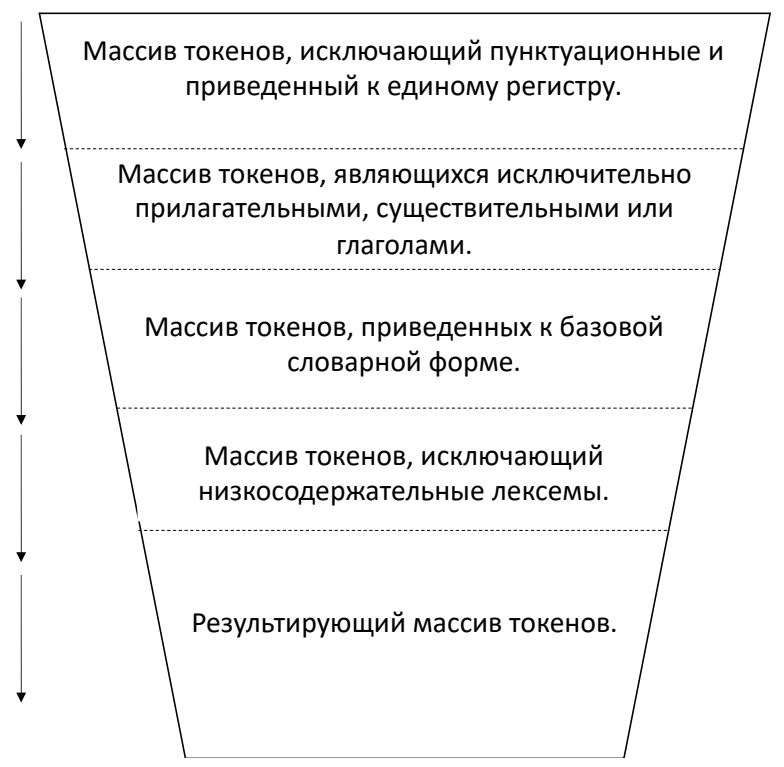

1. Первичная токеннизация входного массива.

2. Морфологическая разметка первичного массива токенов.

3. Лемматизация морфологически размеченного массива токенов.

4. Обработка низкосодержательных лексем.

Рисунок 2. Воронка токенизации.

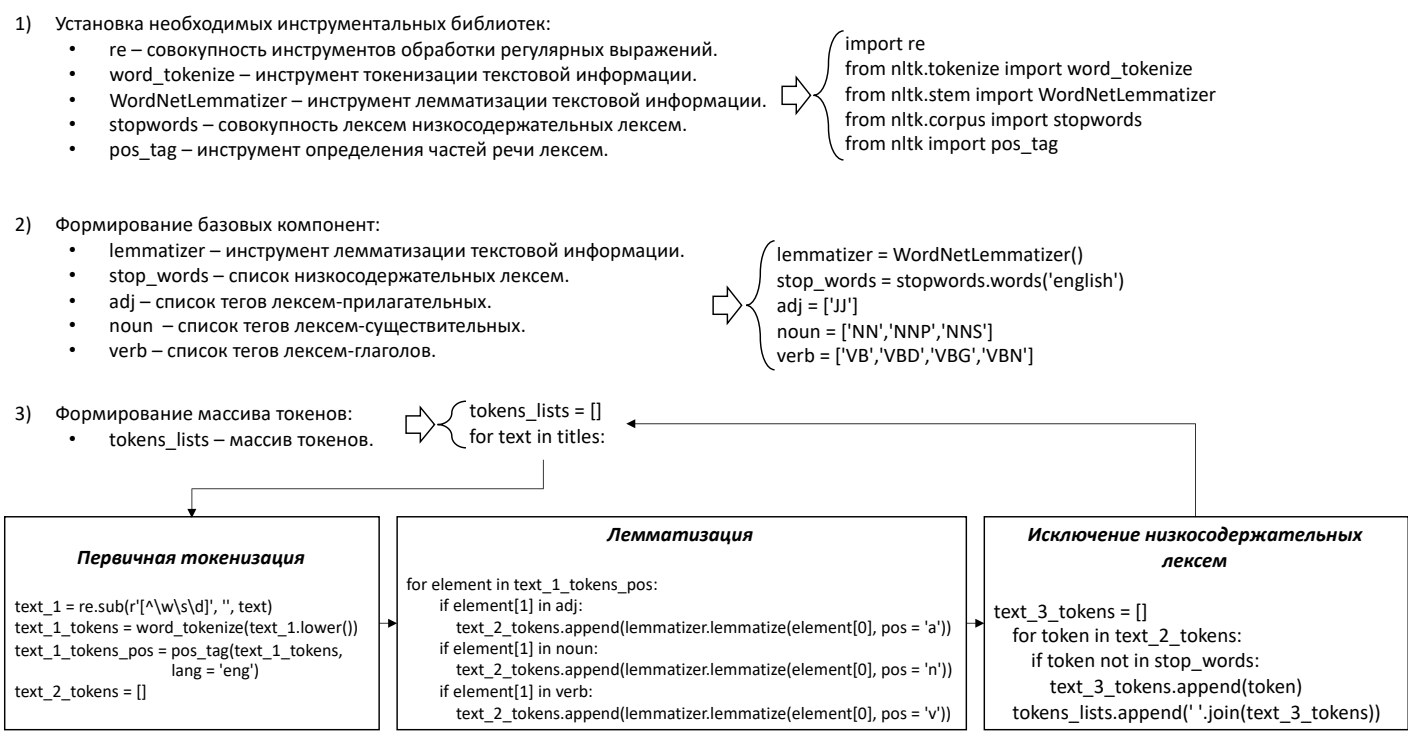

Рисунок 3. Автоматизированный процесс токенизации входного массива текстовой информации. 
сификационного анализа, подразумевающего разбиение исследуемых объектов и признаков на однородные в некотором смысле группы, или кластеры. Исследуемыми объектами в данном случае выступают выделенные ранее токены. Квантификационным признаком выделенных токенов может выступать частота встречаемости того или иного токена в рамках извлеченных ранее заглавий статей. Ключевым дискуссионным вопросом в данном случае является определение оптимального числа выделяемых кластеров. Для целей математической формализации данной идентификации может быть использован показатель силуэтной оценки, отражающий степень подобия точки данных с собственным кластером по сравнению с иными сформированными кластерами [3]. Сущностно, данный показатель отражает качество кластеризации. Таким образом, число кластеров с наибольшей силуэтной оценкой является оптимальным. Детализированный алгоритм идентификации ключевых направлений в рамках исследуемой области представлен на рисунке 4.

Приведенный алгоритм также может быть полностью автоматизирован средствами Python, в частности посредствам библиотеки sklearn. Данная библиотека является одной из наиболее значимых в рамках машинного обучения, и содержит в себе исчерпывающую совокупность инструментов как кластерного анализа, так и оценки качества получаемых результатов. На вход алгоритма кластеризации поступает частотная матрица токенов, именуемая в теории машинного обучения моделью «Word-to-Vek». Результатом кластеризации является совокупность иерархически упорядоченных токенов в рамках каждого из выделенных кластеров. Автоматизированные алгоритм идентификации ключевых направлений в рамках исследуемой области представлен на рисунке 5.

По результатам формирования оптимального числа содержательно-тематических кластеров производится их осмысление. Каждый кластер представляет из себя совокупность упорядоченных токенов, описывающих соответствующее направление в рамках исследуемой области. Однако, токены могут быть дифференцированы токены-центроиды, токены-сателлиты, добавочные токены и случайные токены.

Токены-центроиды определяют содержательно-тематическое ядро кластера, и сущностно являются определяющими. Именно токены-центроиды формируют исключительную смысловую составляющую кластера. Токенысателлиты приобретают осмысленное значение исключительно в сочетании с токенами центроидами. При этом изменение токеновцентроидов определяет изменения значимости и трактовки токенов-сателлитов. Добавочные токены исключительно усиливают и конкретизируют тематику, представленную токенамицентроидами и токенами-сателлитами. Доба-

Массив токеннизированной естественной информации.

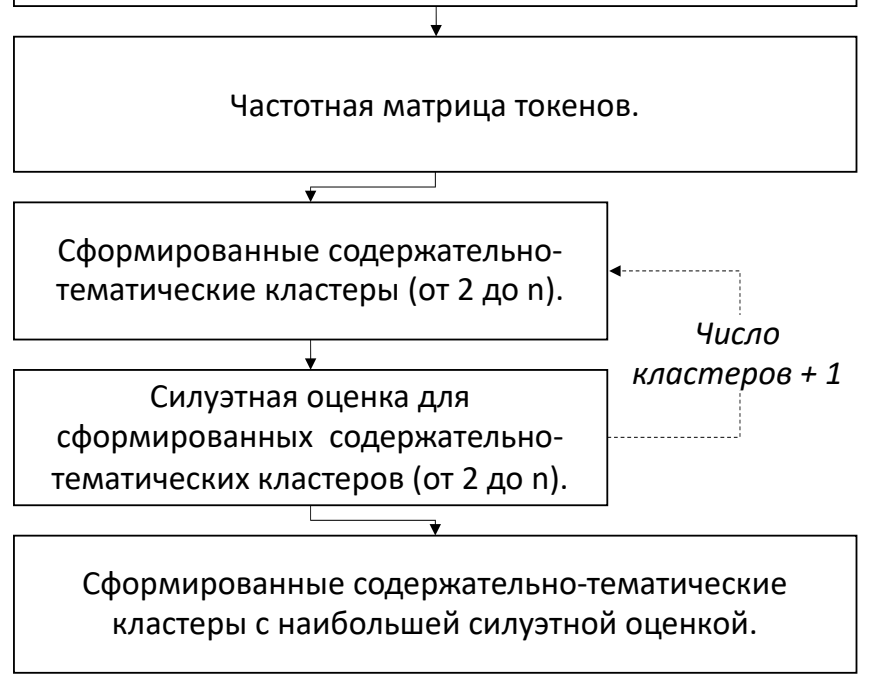

1. Частотная векторизация входного массива.

2. Формирование определенного числа содержательно-тематических кластеров.

3. Расчет силуэтной оценки для заданного числа содержательно-тематических кластеров.

4. Выбор и формирование оптимального числа содержательно-тематических кластеров.

Рисунок 4. Алгоритм идентификации ключевых направлений в рамках исследуемой области. 
1) Установка необходимых инструментальных библиотек:

CountVectorizer - инструмент частотной векторизации массива токенов. _from sklearn.feature_extraction.text import CountVectorizer

- LatentDirichletAllocation - инструмент выделения содержательно- from sklearn.decomposition import LatentDirichletAllocation

- тематических кластеров.
metrics - совокупность инструментов оценки качества кластеризации.

- Kmeans-автоматизированный алгоритм k-средних.

2) Векторизация токеннизированной информации:

X_1 = X_1.toarray()

3) Определение оптимального числа кластеров:

- scores - массив салатных оценок.

- $\quad$ kmeans - модель k-средних для заданного числа кластеров.

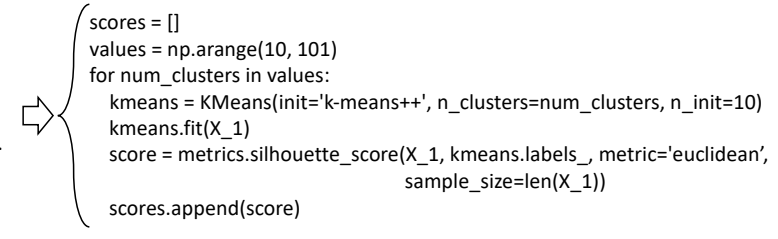

4) Кластеризация входного массива:

- num_clusters - оптимальное число кластеров.

Ida - инструмент выделения содержательнотематических кластеров.

document_topics-кластерное распределение. feature_names - упорядоченная совокупность токенов в рамках каждого из выделенных кластеров.

\section{Рисунок 5. Автоматизированный алгоритм идентификации ключевых направлений} в рамках исследуемой области.

вочные токены, также как и случайные токены в целом не формируют содержательного посыла. Таким образом, процесс осмысления выделенных содержательно-тематических кластеров может быть алгоритмизирован следующим образом:

1. Идентификация токенов-центроидов.

2. Идентификация токенов-сателлитов.

3. Формирование связей между токенами.

4. Формулирование

содержательных токенов-концентраторов.

Содержательные токены-концентраторы представляют собой краткие осмысленные посылы, описывающие содержание каждого из выделенных кластеров. Данные токеныконцентраторы выступают в качестве содержательных запросов к информационной среде. Следовательно, в рамках следующего этапа реализуется процесс автоматизированного извлечения единиц новостной информации. Для целей автоматизированного извлечения единиц новостной информации предлагается использовать инструментарий библиотеки pygooglenews, позволяющий в рамках заданных границ времени извлечь новостные заголовки из базы данных Google, соответствующие сформированным ранее содержательным токенам концентраторам. На рисунке 6 представлен автоматизированный алгоритм извлечения новостных единиц в соответствии с определённым содержательным токеном-концентратором.

В соответствии со сформированным ранее алгоритмом, для целей оценки маркетинговой надежности инновационного решения с точки зрения содержательной компоненты его информационной среды необходимо для каждого идентифицированного направления сформировать массив токенов, описывающих непосредственно маркетинговую надежность. Так как данное свойство является комплексным и многомерным, процесс выделения характеризующих его токенов может быть дифференцирован в соответствии с двумя базовыми подходами:

- Осмысление содержания соответствующего исследуемого направление и экспертное формулирование соответствующих токенов.

- Содержательно-тематическая кластеризация сформированного массива новостных единиц и идентификация кластера токенов, описывающего маркетинговую надежность, в рамках исследуемого направления.

Первый поход подразумевает экспертное осмысление исследуемого направления, что позволяет потенциально получить более значимый массив токенов, исключающий случайные токены. Однако, для целей повсеместной автоматизации предпочтительным является второй подход. На рисунке 7 представлен автоматизированный алгоритм идентификации токенов, отражающих маркетинговую надежность.

Второй и третий этапы данного алгоритма идентичны описанным ранее алгоритмам, представленным на рисунках 3 и 4. Результатам в данном случае выступает оценка присутствия 
1) Установка необходимых инструментальных библиотек:

- GoogleNews - библиотека инструментов извлечения новостных (from pygooglenews import GoogleNews заголовков базы данных сервиса GoogleNews.

- datetime - совокупность инструментов обработки временной информации.

- $\quad$ pandas - совокупность инструментов работы с датафреймами.

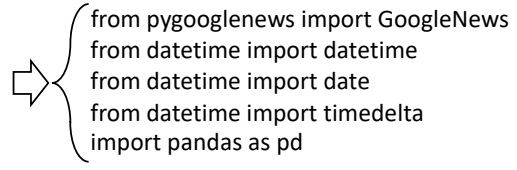

2) Формирование массива временных свойств новостного запроса:

- all_dates_list - массив дат начала новостного запроса.

- date_begin - стартовая дата формирования новостных запросов.

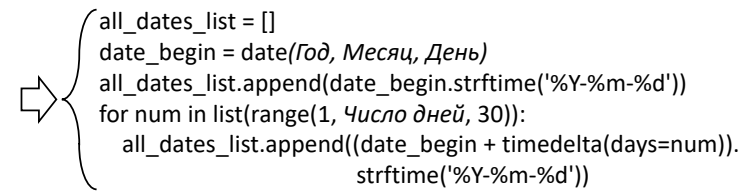

3) Формирование массива новостных единиц:

- gn - свойства извлекаемой новостной информации.

- $\quad$ all_news_list - массив извлеченных новостных единиц.

- news - месячный массив извлеченных новостных единиц.

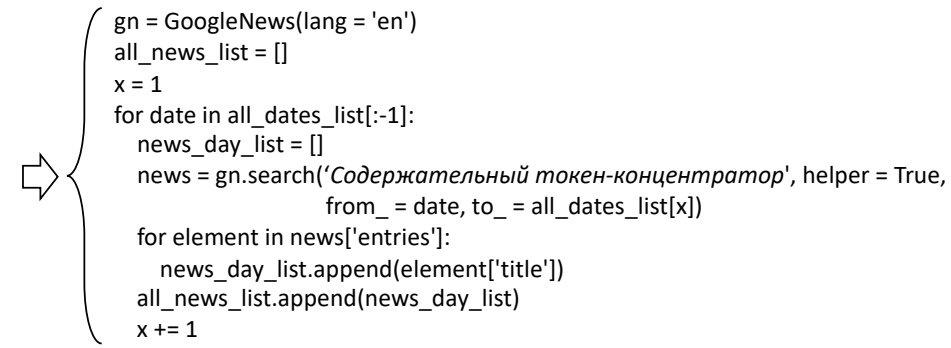

Рисунок 6. Автоматизированный алгоритм извлечения новостных единиц в соответствии с определённым содержательным токеном-концентратором.

Массив содержательных токенов-концентраторов.

Совокупность новостных единиц, соответствующих выделенным токенам-концентраторам.

Массив токенов, описывающих извлеченную совокупность новостных единиц.

Совокупность содержательно-тематических кластеров, описывающих извлеченную совокупность новостных единиц.
1. Извлечение новостных единиц, в соответствии с токенами-концентраторами.

2. Токеннизация извлечённых новостных единиц.

3. Кластеризация массива токенов извлечённых новостных единиц.

Рисунок 7. Автоматизированный алгоритм идентификации токенов, отражающих маркетинговую надежность.

кластера, описывающего маркетинговую надежность в сформированном хронологически структурированном массиве новостных единиц. Оценка уровня маркетинговой надежности производится на основе аппроксимации значений хронологически упорядоченных значений присутствия выделенного кластера линейной функцией, что может быть автоматизировано посредствам библиотеки statsmodels.api. Автоматизированный алгоритм оценки уровня маркетинговой надежности исследуемого на- правления с точки зрения содержательной компоненты его информационной среды представлен на рисунке 8 .

В соответствии со сформированным ранее алгоритмом далее предполагается оценка уровня маркетинговой надежности исследуемого направления с точки зрения тональной компоненты его информационной среды. Данный процесс может быть полностью автоматизирован посредства библиотеки nltk.sentiment.vader, позволяющей оценивать тональные характери- 
1) Установка необходимых инструментальных библиотек:
• statsmodels.арі - совокупность статистических инструментов. $\quad\left\{\begin{array}{l}\text { import statsmodels.api as sm } \\ \text { implats }\end{array}\right.$

2) Оценка уровня маркетинговой надежности с точки зрения содержательной компоненты:

- $\quad X$ - ряд данных, описывающих временной период.

- $\quad \mathrm{Y}$ - показатель присутствия кластера, определяющего маркетинговую надежность, в новостных единицах конкретного временного периода.

- model-сформированная модель регрессии.

- MRC - уровень маркетинговой надежности с точки

зрения содержательной компоненты.

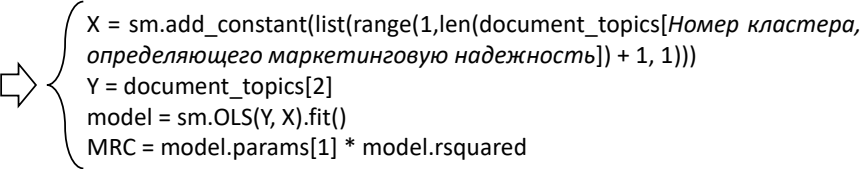

Рисунок 8. Автоматизированный алгоритм оценки уровня маркетинговой надежности исследуемого направления с точки зрения содержательной компоненты его информационной среды.

1) Установка необходимых инструментальных библиотек:

- SentimentIntensityAnalyzer - инструмент оценки тональности.

- numpy-совокупность инструментов работы с векторами.

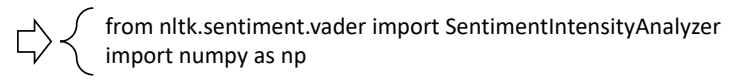

2) Оценка уровня маркетинговой надежности с точки зрения тональной компоненты:

- Nedativ list - массив оценок уровня негативной тональности извлечённых новостных единиц.

- Positiv_list - массив оценок уровня позитивной тональности извлечённых новостных единиц.

- analysis - совокупность оценок тональных характеристик конкретной новостной единицы.

- $\quad$ MRS - уровень маркетинговой надежности с точки зрения тональной компоненты.

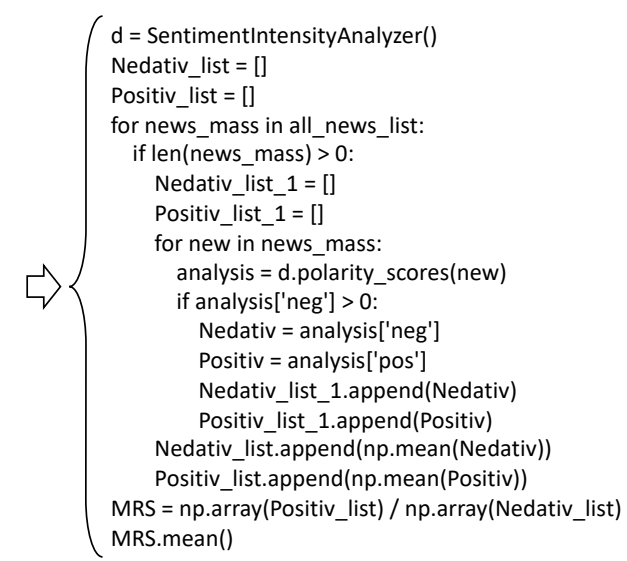

Рисунок 9. Автоматизированный алгоритм оценки уровня маркетинговой надежности исследуемого направления с точки зрения тональной компоненты его информационной среды.

стики текста, представленного на английском языке. Автоматизированный алгоритм оценки уровня маркетинговой надежности исследуемого направления с точки зрения тональной компоненты его информационной среды представлен на рисунке 9.

Полученные оценки уровня маркетинговой надежности исследуемого направления с точки зрения содержательной и тональной компонент его информационной среды нормируются и визуализируются посредствам карты маркетинговой надежности. Таким образом, представленная совокупность инструментов позволяет полностью автоматизировать процесс оценки маркетинговой надежности инновационных решений.

\section{Библиографический список}

1. Родионов Д.Г., Конников Е.А., Куракин В.И. Оценка маркетинговой надежности инновационных решений (на примере нефтепромыслового оборудования) // Экономические науки. 2020. № 193. С. 388-393.

2. Giussani, Bruno. Building the World’s Largest Scientific Database. Газета «The New York Times» (4 марта 1997 года).

3. Родионов Д.Г., Конников Е. А., Конникова О.А. Методология системного анализа информационной среды // Экономические науки. 2021. № 196. С. 160-174.

4. Родионов Д.Г., Конников Е. А., Мугутдинов Р. М. Системный анализ конкурентоспособности цифрового предприятия в рамках информационной среды // Экономические науки. 2020. № 193. С. 394-401.

5. Родионов Д.Г., Ялымов С. В., Конников Е. А. Влияние информационной среды на субъекты малого и среднего предпринимательства // Экономические науки. 2020. № 189. С. 86-91. 